\title{
On Solutions of Linear Fractional Differential Equations with Uncertainty
}

\author{
T. Allahviranloo, ${ }^{1}$ S. Abbasbandy, ${ }^{2}$ M. R. Balooch Shahryari, ${ }^{2}$ \\ S. Salahshour, ${ }^{3}$ and D. Baleanu ${ }^{4,5,6}$ \\ ${ }^{1}$ Department of Electronic and Communications, Faculty of Engineering, Izmir University, Izmir, Turkey \\ ${ }^{2}$ Department of Mathematics, Science and Research Branch, Islamic Azad University, Tehran, Iran \\ ${ }^{3}$ Young Researchers and Elite Club, Mobarakeh Branch, Islamic Azad University, Mobarakeh, Iran \\ ${ }^{4}$ Department of Chemical and Materials Engineering, Faculty of Engineering, King Abdulaziz University, Jeddah 21589, Saudi Arabia \\ ${ }^{5}$ Department of Mathematics and Computer Sciences, Faculty of Arts and Sciences, Cankaya University, 06530 Ankara, Turkey \\ ${ }^{6}$ Institute of Space Sciences, Magurele-Bucharest, Bucharest, RO 7690, Romania
}

Correspondence should be addressed to S. Salahshour; soheilsalahshour@yahoo.com

Received 8 June 2013; Revised 29 August 2013; Accepted 30 August 2013

Academic Editor: Ali H. Bhrawy

Copyright (C) 2013 T. Allahviranloo et al. This is an open access article distributed under the Creative Commons Attribution License, which permits unrestricted use, distribution, and reproduction in any medium, provided the original work is properly cited.

The solutions of linear fuzzy fractional differential equations (FFDEs) under the Caputo differentiability have been investigated. To this end, the fuzzy Laplace transform was used to obtain the solutions of FFDEs. Then, some new results regarding the relation between some types of differentiability have been obtained. Finally, some applicable examples are solved in order to show the ability of the proposed method.

\section{Introduction}

The topic of fuzzy differential equations (FDEs) has been rapidly growing in recent years (see, e.g., $[1-8]$ and the references therein). Recently, applying fractional differential equations has grown increasingly and have excited as a considerable interest both in mathematics and applications, such that they were used in modeling of many physical and chemical processes and in engineering [9-15].

In addition, many research papers are published to consider solutions of fractional differential equations (see, e.g., $[16-18]$ and the references therein).

Recently, Agarwal et al. [19] proposed the concept of solutions for fractional differential equations with uncertainty. They considered the Riemann-Liouville differentiability to solve FFDEs which is a combination of Hukuhara difference and Riemann-Liouville derivative. There are some basic papers which are written by Bede and Gal [20] who discussed on shortcomings of applications of Hukuhara difference.
So, we adopt a generalization of a strongly generalized differentiability to a fractional case.

In the following, we propose the Riemann-Liouville differentiability by using Hukuhara difference which is named as the Riemann-Liouville H-differentiability. Therefore, a direct procedure is adopted to derive such concept which is constructed based on the combination of strongly generalized differentiability [21] and the Riemann-Liouville derivative $[14,15]$. Moreover, we suggest the concept of fractional derivatives under Caputo's differentiability by applying the Hukuhara difference which is named as Caputo's $\mathrm{H}-$ differentiability. Similar to the deterministic cases, construction of Caputo's derivatives is based on the definitions of the Riemann-Liouville derivatives in fuzzy cases.

Consequently, we intend to propose an analytical method to solve FFDEs. Since, considering solutions of FFDEs is a new subject, presets the fractional Green's functions for fuzzy fractional differential equations is considered and, as particular cases, we obtain the classical harmonic oscillator, 
the damped harmonic oscillator, relaxation equation, all in the fuzzy fractional versions by using fuzzy laplace transforms; so we should first implement analytical method to solve it; then numerical methods can be applied. To this end, we adopt fractional Green's functions to solve FFDEs by using fuzzy Laplace transforms method. One can see some useful papers about fuzzy Laplace transforms in [2, 22, 23].

Recently, Salahshour et al. proposed some new results toward existence and uniqueness of the solutions of FFDE [24]. Then, Mazandarani and Kamyad numerically solved the FFDE using the Euler method [25]. Also, Agarwal et al. [26] investigated fuzzy fractional integral equation under compactness type condition.

This paper is structured as follows. In Section 1, we recall some well-known definitions of fuzzy numbers and present some needed concepts. In Section 2, Caputo's Hdifferentiability is introduced, and the relation between the Riemann-Liouville differentiability and Caputo's $\mathrm{H}$ differentiability and some of their properties is considered. Consequently, the fuzzy Laplace transforms are considered for fuzzy-valued function, and an essential theorem for the Laplace transform of $f$ under Caputo's H-derivative is given in Section 3. The solutions of FFDEs are investigated by using the fuzzy Laplace transforms and their inverses in Section 4. In Section 5, some examples are solved to illustrate the method. Finally, conclusion is drawn in Section 6.

\section{Preliminaries}

The basic definition of fuzzy numbers can be seen in [27].

We denote by $\mathbb{R}$ the set of all real numbers and the set of all fuzzy numbers on $\mathbb{R}$ is indicated by $\mathbb{E}$. A fuzzy number is a mapping $u: \mathbb{R} \rightarrow[0,1]$ with the following properties.

(a) $u$ is upper semicontinuous.

(b) $u$ is fuzzy convex; that is, $u(\lambda x+(1-\lambda) y) \geq$ $\min \{u(x), u(y)\}$ for all $x, y \in \mathbb{R}, \lambda \in[0,1]$.

(c) $u$ is normal; that is; $\exists x_{0} \in \mathbb{R}$ for which $u\left(x_{0}\right)=1$.

(d) supp $u=\{x \in \mathbb{R} \mid u(x)>0\}$ is the support of the $u$, and its closure $\mathrm{cl}(\operatorname{supp} u)$ is compact.

An equivalent parametric definition is also given in [2830] as follows.

Definition 1. A fuzzy number $u$ in parametric form is a pair $(\underline{u}, \bar{u})$ of functions $\underline{u}(r), \bar{u}(r), 0 \leq r \leq 1$, which satisfy the following requirements:

(1) $\underline{u}(r)$ is a bounded nondecreasing left continuous function in $(0,1]$; and right continuous at 0 ;

(2) $\bar{u}(r)$ is a bounded nonincreasing left continuous function in $(0,1]$; and right continuous at 0 ;

(3) $\underline{u}(r) \leq \bar{u}(r), 0 \leq r \leq 1$.

Moreover, we also can present the $r$-cut representation of fuzzy number as $[u]^{r}=[\underline{u}(r), \bar{u}(r)]$ for all $0 \leq r \leq 1$.
According to Zadeh's extension principle, operation of addition on $\mathbb{E}$ is defined by

$$
(u \oplus v)(x)=\sup _{y \in \mathbb{R}} \min \{u(y), v(x-y)\}, \quad x \in \mathbb{R},
$$

and scalar multiplication of a fuzzy number is given by

$$
(k \odot u)(x)= \begin{cases}u\left(\frac{x}{k}\right), & k>0, \\ \tilde{0}, & k=0,\end{cases}
$$

where $\widetilde{0} \in \mathbb{E}$.

The Hausdorff distance between fuzzy numbers is given by $d: \mathbb{E} \times \mathbb{E} \rightarrow \mathbb{R}_{+} \cup\{0\}$,

$$
d(u, v)=\sup _{r \in[0,1]} \max \{|\underline{u}(r)-\underline{v}(r)|,|\bar{u}(r)-\bar{v}(\alpha)|\},
$$

where $u=(\underline{u}(r), \bar{u}(r)), v=(\underline{v}(r), \bar{v}(r)) \subset \mathbb{R}$ is utilized in [20]. Then, it is easy to see that $\bar{d}$ is a metric in $\mathbb{E}$ and has the following properties (see [31]):

(1) $d(u+w, v+w)=d(u, v)$, for all $u, v, w \in \mathbb{E}$,

(2) $d(k u, k v)=|k| d(u, v)$, for all $k \in \mathbb{R}, u, v \in \mathbb{E}$,

(3) $d(u+v, w+e) \leq d(u, w)+d(v, e)$, for all $u, v, w, e \in \mathbb{E}$,

(4) $(d, \mathbb{E})$ is a complete metric space.

Theorem 2 (see [32]). Let $f(x)$ be a fuzzy-valued function on $[a, \infty)$, and it is represented by $(\underline{f}(x ; r), \bar{f}(x ; r))$. For any fixed $r \in[0,1]$, assume that $f(x ; r)$ and $\bar{f}(x ; r)$ are Riemannintegrable on $[a, b]$ for every $b \geq a$, and assume that there are two positive $\underline{M}(r)$ and $\bar{M}(r)$ such that $\int_{a}^{b}|\underline{f}(x ; r)| d x \leq$ $\underline{M}(r)$ and $\int_{a}^{b}|\bar{f}(x ; r)| d x \leq \bar{M}(r)$ for every $b \geq a$. Then $f(x)$ is improper fuzzy Riemann-integrable on $[a, \infty)$, and the improper fuzzy Riemann-integral is a fuzzy number. Furthermore, one has:

$$
\int_{a}^{\infty} f(x) d x=\left(\int_{a}^{\infty} \underline{f}(x ; r) d x, \int_{a}^{\infty} \bar{f}(x ; r) d x\right) .
$$

Definition 3. Let $x, y \in \mathbb{E}$. If there exists $z \in \mathbb{E}$ such that $x=y+z$, then $z$ is called the H-difference of $x$ and $y$, and it is denoted by $x \ominus y$.

In this paper, the sign " $\ominus$ " always stands for $\mathrm{H}$-difference, and also note that $x \ominus y \neq x+(-1) y$.

2.1. Caputo's H-Differentiability. In this section, the concept of the fuzzy Caputo derivatives has been reviewed [3335]. Also, we denote by $C^{\mathbb{F}}[a, b]$ a space of all fuzzy-valued functions which are continuous on $[a, b]$. Also, we denote the space of all Lebesgue integrable fuzzy-value functions on the bounded interval $[a, b] \subset \mathbb{R}$ by $L^{\mathbb{F}}[a, b]$, and we denote the space of fuzzy-value functions $f(x)$ which have continuous $\mathrm{H}$-derivative up to order $n-1$ on $[a, b]$ such that $f^{(n-1)}(x) \in$ $A C^{\mathbb{F}}([a, b])$ by $A C^{(n) \mathbb{F}}([a, b])$. 
Definition 4. Let $f \in C^{\mathbb{F}}[a, b] \cap L^{\mathbb{F}}[a, b]$; the fuzzy RiemannLiouville integral of fuzzy-valued function $f$ is defined as follows:

$$
\left(I_{a^{+}}^{\beta} f\right)(x)=\frac{1}{\Gamma(\beta)} \int_{a}^{x} \frac{f(t) d t}{(x-t)^{1-\beta}}, \quad x>a, 0<\beta<1 .
$$

Since $f(x ; r)=[f(x ; r), \bar{f}(x ; r)]$, for all $0 \leq r \leq 1$, then one can indicate the fuzzy Riemann-Liouville integral of fuzzyvalued function $f$ based on the lower and upper functions as follows.

Theorem 5. Let $f(x) \in C^{\mathbb{F}}[a, b] \cap L^{\mathbb{F}}[a, b]$; the fuzzy Riemann-Liouville integral of fuzzy-valued function $f$ is defined by

$$
\left(I_{a^{+}}^{\beta} f\right)(x ; r)=\left[\left(I_{a^{+}}^{\beta} \underline{f}\right)(x ; r),\left(I_{a^{+}}^{\beta} \bar{f}\right)(x ; r)\right], \quad 0 \leq r \leq 1,
$$

where

$$
\begin{array}{ll}
\left(I_{a^{+}}^{\beta} \underline{f}\right)(x ; r)=\frac{1}{\Gamma(\beta)} \int_{a}^{x} \frac{\frac{f(t ; r) d t}{(x-t)^{1-\beta}},}{}, \quad 0 \leq r \leq 1, \\
\left(I_{a^{+}}^{\beta \bar{f}}\right)(x ; r)=\frac{1}{\Gamma(\beta)} \int_{a}^{x} \frac{\bar{f}(t ; r) d t}{(x-t)^{1-\beta}}, \quad 0 \leq r \leq 1 .
\end{array}
$$

Now, we define the fuzzy Riemann-Liouville fractional derivatives of order $n-1<\beta<n$ for fuzzy-valued function $f$ (which is a direct extension of strongly generalized $\mathrm{H}$ differentiability [20] and lateral type of H-differentiability [36] in the fractional literature) as follows.

Definition 6. Let $f^{n} \in C^{(n) \mathbb{F}}[a, b] \cap L_{1}^{\mathbb{F}}[a, b]$, let $x_{0} \in(a, b)$, and let $\Phi(x)=(1 / \Gamma(n-\beta)) \int_{a}^{x}\left(f(t) d t /(x-t)^{\beta-n+1}\right)(n=$ $[\beta]+1 ; x>a)$. We say that $f$ is fuzzy Riemann-Liouville fractional differentiable of $\operatorname{order} \beta$, at $x_{0}$, if there exists an element $\left(D_{a^{+}}^{\beta} f\right)\left(x_{0}\right) \in \mathbb{E}$, such that for all $h>0$ sufficiently small

(i)

$$
\begin{aligned}
\left(D_{a^{+}}^{\beta} f\right)\left(x_{0}\right) & =\lim _{h \rightarrow 0} \frac{\Phi^{(n-1)}\left(x_{0}+h\right) \ominus \Phi^{(n-1)}\left(x_{0}\right)}{h} \\
& =\lim _{h \rightarrow 0} \frac{\Phi^{(n-1)}\left(x_{0}\right) \ominus \Phi^{(n-1)}\left(x_{0}-h\right)}{h}
\end{aligned}
$$

or

(ii)

$$
\begin{aligned}
\left(D_{a^{+}}^{\beta} f\right)\left(x_{0}\right) & =\lim _{h \rightarrow 0} \frac{\Phi^{(n-1)}\left(x_{0}\right) \ominus \Phi^{(n-1)}\left(x_{0}+h\right)}{-h} \\
& =\lim _{h \rightarrow 0} \frac{\Phi^{(n-1)}\left(x_{0}-h\right) \ominus \Phi^{(n-1)}\left(x_{0}\right)}{-h} .
\end{aligned}
$$

For sake of simplicity, we say that a fuzzy-valued function $f$ is $R L[(i)-\beta]$-differentiable if it is differentiable as in Definition 6 case (i) and is ${ }^{R L}[(\mathrm{ii})-\beta]$-differentiable if it is differentiable as in Definition 6 case (ii).
Theorem 7. Let $f(x) \in C^{(n) \mathbb{F}}[a, b] \cap L^{\mathbb{F}}[a, b], x_{0} \in(a, b)$, $(\beta>0)$, and $(n=[\beta]+1)$ such that for all $0 \leq r \leq 1$, then

(i) if $f(x)$ is a ${ }^{R L}[(i)-\beta]$-differentiable fuzzy-valued function, then

$$
\left({ }^{R L} D_{a^{+}}^{\beta} f\right)\left(x_{0} ; r\right)=\left[{ }^{R L} D_{a^{+}}^{\beta} \underline{f}\left(x_{0}, r\right),{ }^{R L} D_{a^{+}}^{\beta} \bar{f}\left(x_{0}, r\right)\right] ;
$$

(ii) if $f(x)$ is $a^{R L}[(i i)-\beta]$-differentiable fuzzy-valued function, then

$$
\left({ }^{R L} D_{a^{+}}^{\beta} f\right)\left(x_{0} ; r\right)=\left[{ }^{R L} D_{a^{+}}^{\beta \bar{f}}\left(x_{0} ; r\right),{ }^{R L} D_{a^{+}}^{\beta} \underline{f}\left(x_{0}, r\right)\right],
$$

where

$$
\begin{aligned}
& \left({ }^{R L} D_{a^{+}}^{\beta}, f\right)\left(x_{0} ; r\right)=\left[\frac{1}{\Gamma(n-\beta)} \frac{d^{n}}{d x^{n}} \int_{a}^{x} \frac{\underline{f}(t ; r) d t}{(x-t)^{\beta-n+1}}\right]_{x=x_{0}}, \\
& \left({ }^{R L} D_{a^{+}}^{\beta-}\right)\left(x_{0} ; r\right)=\left[\frac{1}{\Gamma(n-\beta)} \frac{d^{n}}{d x^{n}} \int_{a}^{x} \frac{\bar{f}(t, r) d t}{(x-t)^{\beta-n+1}}\right]_{x=x_{0}} .
\end{aligned}
$$

Definition 8. Let $f \in C^{(n) \mathbb{F}}[a, b] \cap L_{1}^{\mathbb{F}}[a, b]$, and $f^{(n)}$ is integrable; then the right fuzzy Caputo derivative of $f$ for $n-1<\beta<n$ and $x \in[a, b]$ is denoted by $\left({ }^{C} D_{a^{+}}^{\beta} f\right)(x) \in \mathbb{E}$ and defined by

$$
\left({ }^{C} D_{a^{+}}^{\beta} f\right)(x)=\frac{1}{\Gamma(n-\beta)} \odot \int_{a}^{x}(x-t)^{-\beta+n-1} \odot f^{(n)}(t) d t .
$$

Theorem 9. Let $f(x) \in C^{(n) \mathbb{F}}[a, b] \cap L^{\mathbb{F}}[a, b], x \in(a, b)$, and $n-1<\beta<n$, such that for all $0 \leq r \leq 1$, then then

(i) if $f$ is $a^{C}[(i)-\beta]$-differentiable fuzzy-valued function,

$$
\left({ }^{C} D_{a^{+}}^{\beta} f\right)(x ; r)=\left[\left({ }^{C} D_{a^{+}}^{\beta} \underline{f}\right)(x ; r),\left({ }^{C} D_{a^{+}}^{\beta} \bar{f}\right)(x ; r)\right] ;
$$

(ii) if $f(x)$ is a ${ }^{C}[(i i)-\beta]$-differentiable fuzzy-valued function, then

$$
\left({ }^{C} D_{a^{+}}^{\beta} f\right)(x ; r)=\left[\left({ }^{C} D_{a^{+}}^{\beta} \bar{f}\right)(x ; r),\left({ }^{C} D_{a^{+}}^{\beta} \underline{f}\right)(x ; r)\right],
$$


where

$$
\begin{aligned}
\left({ }^{C} D_{a^{+}}^{\beta} \underline{f}\right)(x ; r) & { }^{R L} D_{a^{+}}^{\beta}\left[\underline{f}(t ; r)-\sum_{k=0}^{n-1} \frac{x^{k}}{k !} \underline{f}^{(k)}(a ; r)\right](x ; r) \\
= & \frac{1}{\Gamma(n-\beta)} \frac{d^{n}}{d x^{n}} \\
& \times \int_{a}^{x} \frac{\underline{f}(t ; r)-\sum_{k=0}^{n-1}\left(t^{k} / k !\right) \underline{f}^{(k)}(a ; r)}{(x-t)^{\beta-n+1}} d t, \\
\left.{ }^{C} D_{a^{+}}^{\beta} \bar{f}\right)(x ; r) & { }^{R L} D_{a^{+}}^{\beta}\left[\bar{f}(t ; r)-\sum_{k=0}^{n-1} \frac{x^{k}}{k !} \bar{f}^{(k)}(a ; r)\right](x ; r) \\
= & \frac{1}{\Gamma(n-\beta)} \frac{d^{n}}{d x^{n}} \\
& \times \int_{a}^{x} \frac{\bar{f}(t, r)-\sum_{k=0}^{n-1}\left(t^{k} / k !\right) \bar{f}(k)}{(x-t)^{\beta-n+1}} d t .
\end{aligned}
$$

Proof. We prove the case of ${ }^{C}[(\mathrm{i})-\beta]$-differentiability, $(\beta)>$ $0,(n=[\beta]+1)$, and for the case of ${ }^{C}[(\mathrm{ii})-\beta]$-differentiability the proof is completely similar to previous one, and hence it is omitted.

Let us consider that $f$ is ${ }^{C}[(\mathrm{i})-\beta]$-differentiability and that $x \in(a, b)$; then we have the following:

$$
\begin{aligned}
& {\left[\Phi^{(n-1)}(x+h) \ominus \Phi^{(n-1)(x)}\right]^{r}} \\
& =\left[\underline{\Phi}^{(n-1)}(x+h ; r)\right. \\
& \left.\quad-\underline{\Phi}^{(n-1)}(x ; r), \bar{\Phi}^{(n-1)}(x+h ; r)-\bar{\Phi}^{(n-1)}(x ; r)\right],
\end{aligned}
$$

and multiplying by $1 / h$, we get the following:

$$
\begin{aligned}
\frac{\left[\Phi^{(n-1)}(x+h) \ominus \Phi^{(n-1)}(x)\right]^{r}}{h} \\
=\left[\frac{\Phi^{(n-1)}(x+h ; r)-\underline{\Phi}^{(n-1)}(x ; r)}{h},\right. \\
\\
\left.\frac{\bar{\Phi}^{(n-1)}(x+h ; r)-\Phi^{(n-1)}(x ; r)}{h}\right] .
\end{aligned}
$$

Similarly, we get the following:

$$
\begin{aligned}
& \frac{\left[\Phi^{(n-1)}(x) \ominus \Phi^{(n-1)}(x-h)\right]^{r}}{h} \\
&=\left[\frac{\underline{\Phi}^{(n-1)}(x ; r)-\underline{\Phi}^{(n-1)}(x-h ; r)}{h},\right. \\
&\left.\frac{\bar{\Phi}^{(n-1)}(x ; r)-\bar{\Phi}^{(n-1)}(x-h ; r)}{h}\right] .
\end{aligned}
$$

Passing to the limit we obtain

$$
\left({ }^{C} D_{a^{+}}^{\beta} f\right)(x ; r)=\left[{ }^{C} D_{a^{+}}^{\beta} \underline{f}(x ; r),{ }^{C} D_{a^{+}}^{\beta} \bar{f}(x ; r)\right],
$$

which proves the theorem.

\section{The Fuzzy Laplace Transforms}

In this section, we consider the fuzzy Laplace transform for fuzzy-valued function; then derivative theorem is given which is essential to determine solutions of FFDEs.

In this way, Allahviranloo and Ahmadi [2] suggested the concept of Laplace transforms for fuzzy-valued function as follows.

Definition 10 (see [2]). Let $f(x)$ be continuous fuzzy-value function. Suppose that $f(x) \odot e^{-p x}$ is improper fuzzy Riemann-integrable on $[0, \infty)$; then $\int_{0}^{\infty} f(x) \odot e^{-p x} d x$ is called fuzzy Laplace transforms and is denoted by

$$
\mathbf{L}[f(x)]=\int_{0}^{\infty} f(x) \odot e^{-p x} d x \quad(p>0 \text { and integer }) .
$$

Remark 11. In [8], the authors have investigated under what conditions the fuzzy-valued functions can possess the fuzzy Laplace transform. So, we suppose that the given fuzzyvalued functions have mentioned conditions throughout the paper.

From Theorem 2, we have

$$
\begin{aligned}
\int_{0}^{\infty} f & (x) \odot e^{-p x} d x \\
& =\left(\int_{0}^{\infty} \underline{f}(x ; r) e^{-p x} d x, \int_{0}^{\infty} \bar{f}(x ; r) e^{-p x} d x\right) .
\end{aligned}
$$

Also by using the definition of classical Laplace transform one has

$$
\begin{aligned}
& \ell[\underline{f}(x ; r)]=\int_{0}^{\infty} \underline{f}(x ; r) e^{-p x} d x, \\
& \ell[\bar{f}(x, r)]=\int_{0}^{\infty} \bar{f}(x ; r) e^{-p x} d x ;
\end{aligned}
$$

then we get

$$
\mathbf{L}[f(x)]=(\ell[\underline{f}(x ; r)], \ell[\bar{f}(x ; r)]) .
$$


Theorem 12 (see [2]). Let $f(x), g(x)$ be continuous-fuzzyvalued functions; suppose that $c_{1}, c_{2}$ are constant; then

$$
\begin{aligned}
\mathbf{L} & {\left[\left(c_{1} \odot f(x)\right) \oplus\left(c_{2} \odot g(x)\right)\right] } \\
& =\left(c_{1} \odot \mathbf{L}[f(x)]\right) \oplus\left(c_{2} \odot \mathbf{L}[g(x)]\right) .
\end{aligned}
$$

Lemma 13 (see [2]). Let $f(x)$ be continuous fuzzy-value function on $[0, \infty)$ and let $\lambda \in \mathbb{R}$; then

$$
\mathbf{L}[\lambda \odot f(x)]=\lambda \odot \mathbf{L}[f(x)] .
$$

Lemma 14 (see [2]). Let $f(x)$ be continuous fuzzy-value function and $g(x) \geq 0$. Suppose that $(f(x) \odot g(x)) \odot e^{-p x}$ is improper fuzzy Riemann-integrable on $[0, \infty)$; then

$$
\begin{aligned}
& \int_{0}^{\infty}(f(x) \odot g(x)) \odot e^{-p x} d x \\
& \quad=\left(\int_{0}^{\infty} g(x) \underline{f}(x, r) e^{-p x} d x, \int_{0}^{\infty} g(x) \bar{f}(x, r) e^{-p x} d x\right) .
\end{aligned}
$$

Theorem 15 (first translation theorem (see [2])). Let $f$ be continuous fuzzy value function and let $\mathbf{L}[f(x)]=F(p)$. Then

$$
\mathbf{L}\left[e^{a x} \odot f(x)\right]=F(p-a),
$$

where $e^{a x}$ is real value function.

In order to solve FFDEs, it is necessary to know the fuzzy Laplace transform of Caputo's H-derivative of $f,\left({ }^{C} D_{a^{+}}^{\beta} f\right)(x)$. The virtue of $\mathbf{L}\left[\left({ }^{C} D_{a^{+}}^{\beta} f\right)(x)\right]$ is that it can be written in terms of $\mathbf{L}[f(x)]$.

Theorem 16 (derivative theorem). Suppose that $f(x) \in$ $C^{(n) \mathbb{F}}[0, \infty) \cap L^{\mathbb{E}}[0, \infty)$; then

$$
\begin{aligned}
\mathbf{L}\left[\left({ }^{C} D_{a^{+}}^{\beta} f\right)(x)\right] \\
\quad=p^{\beta} \mathbf{L}[f(t)] \ominus\left(\sum_{k=0}^{n-1} p^{\beta-k-1} f^{(k)}\right)(0)
\end{aligned}
$$

if $D^{(k-1)} f$ is ${ }^{C}[(i)-\beta]$-differentiable, and also

$$
\begin{aligned}
\mathbf{L}\left[\left({ }^{C} D_{a^{+}}^{\beta} f\right)(x)\right] \\
\quad=-\left(\sum_{k=0}^{n-1} p^{\beta-k-1} f^{(k)}\right)(0) \ominus\left(-p^{\beta} \mathbf{L}[f(t)]\right)
\end{aligned}
$$

if $D^{(k-1)} f$ is ${ }^{C}[(i i)-\beta]$-differentiable.

Proof. For arbitrary fixed $r \in[0,1]$ we have

$$
\begin{aligned}
p^{\beta} \mathbf{L}[f(t)] \ominus p^{\beta-1} f(0) & \\
= & {\left[p^{\beta} \ell[\underline{f}(x ; r)]-\left(\sum_{k=0}^{n-1} p^{\beta-k-1} \underline{f}^{(k)}\right)(0 ; r),\right.} \\
& \left.p^{\beta} \ell[\bar{f}(x ; r)]-\left(\sum_{k=0}^{n-1} p^{\beta-k-1} \bar{f}^{(k)}\right)(0 ; r)\right] .
\end{aligned}
$$

Since $D^{(k-1)} f$ is ${ }^{C}[(\mathrm{i})-\beta]$, we get the following:

$$
\begin{aligned}
\left({ }^{C} D_{a^{+}}^{\beta} f\right)(x ; r) & =\left[\left({ }^{C} D_{a^{+}}^{\beta} f\right)(x ; r),\left(\overline{{ }^{C} D_{a^{+}}^{\beta} f}\right)(x ; r)\right] \\
& =\left[\left({ }^{C} D_{a^{+}}^{\beta} f\right)(x ; r),\left({ }^{C} D_{a^{+}}^{\beta} \bar{f}\right)(x ; r)\right] .
\end{aligned}
$$

Hence, we get

$$
\begin{aligned}
\ell\left[\left(\overline{{ }^{C} D_{a^{+}}^{\beta} f}\right)(x ; r)\right]= & \ell\left[\left({ }^{C} D_{a^{+}}^{\beta} \bar{f}\right)(x ; r)\right] \\
= & p^{\beta} \ell[\bar{f}(x ; r)] \\
& -\left(\sum_{k=0}^{n-1} p^{\beta-k-1} \bar{f}^{(k)}\right)(0 ; r), \\
\ell\left[\left({ }^{C} D_{a^{+}}^{\beta} f\right)(x ; r)\right]= & \ell\left[\left({ }^{C} D_{a^{+}}^{\beta} \underline{f}\right)(x ; r)\right] \\
= & p^{\beta} \ell[\underline{f}(x ; r)] \\
& -\left(\sum_{k=0}^{n-1} p^{\beta-k-1} \underline{f}^{(k)}\right)(0 ; r) .
\end{aligned}
$$

Then, we obtained the following:

$$
\begin{aligned}
p^{\beta} \mathbf{L} & {[f(x)] \ominus\left(\sum_{k=0}^{n-1} p^{\beta-k-1} f^{(k)}\right)(0) } \\
& =\left(\ell\left[\left({ }^{C} D_{a^{+}}^{\beta} \underline{f}\right)(x ; r)\right], \ell\left[\left({ }^{C} D_{a^{+}}^{\beta} \bar{f}\right)(x ; r)\right]\right) .
\end{aligned}
$$

Then, we have

$$
\begin{aligned}
p^{\beta} \mathbf{L} & {[f(x)] \ominus\left(\sum_{k=0}^{n-1} p^{\beta-k-1} f^{(k)}\right)(0) } \\
& =\mathbf{L}\left[\left({ }^{C} D_{a^{+}}^{\beta} \underline{f}\right)(x ; r),\left({ }^{C} D_{a^{+}}^{\beta} \bar{f}\right)(x ; r)\right] .
\end{aligned}
$$

Using (32) leads to obtain

$$
p^{\beta} \mathbf{L}[f(x)] \ominus\left(\sum_{k=0}^{n-1} p^{\beta-k-1} f^{(k)}\right)(0)=\mathbf{L}\left[\left({ }^{C} D_{a^{+}}^{\beta} f\right)(x)\right] .
$$


Now, we assume that $D^{(k-1)} f$ is the ${ }^{C}[(\mathrm{ii})-\beta]$; then for arbitrary fixed $r \in[0,1]$ we have

$$
\begin{gathered}
-\left(\sum_{k=0}^{n-1} p^{\beta-k-1} f^{(k)}\right)(0) \ominus\left(-p^{\beta} \mathbf{L}[f(t)]\right) \\
=\left(-f_{2}(t ; r),-f_{1}(t ; r)\right), \\
-f_{2}(t ; r)=-\left(\sum_{k=0}^{n-1} p^{\beta-k-1} \bar{f}^{(k)}\right)(0 ; r)+p^{\beta} \ell[\bar{f}(x ; r)], \\
-f_{1}(t ; r)=-\left(\sum_{k=0}^{n-1} p^{\beta-k-1} \underline{f}^{(k)}\right)(0 ; r)+p^{\beta} \ell[\underline{f}(x ; r)] .
\end{gathered}
$$

Since $D^{(k-1)} f$ is ${ }^{C}[(i i)-\beta]$, we get

$$
\begin{aligned}
\left({ }^{C} D_{a^{+}}^{\beta} f\right)(x ; r) & =\left[\left({ }^{C} D_{a^{+}}^{\beta} f\right)(x ; r),\left(\overline{{ }^{C} D_{a^{+}}^{\beta} f}\right)(x ; r)\right] \\
& =\left[\left({ }^{C} D_{a^{+}}^{\beta} \bar{f}\right)(x ; r),\left({ }^{C} D_{a^{+}}^{\beta} f\right)(x ; r)\right],
\end{aligned}
$$

and, hence, we get

$$
\begin{aligned}
- & \left(\sum_{k=0}^{n-1} p^{\beta-k-1} f^{(k)}\right)(0) \ominus\left(-p^{\beta} \mathbf{L}[f(t)]\right) \\
= & p^{\beta} \ell[\bar{f}(x ; r)]-\left(\sum_{k=0}^{n-1} p^{\beta-k-1} \bar{f}^{(k)}\right)(0 ; r), p^{\beta} \ell[\underline{f}(x ; r)] \\
& -\left(\sum_{k=0}^{n-1} p^{\beta-k-1} \underline{f}^{(k)}\right)(0 ; r) .
\end{aligned}
$$

So, we get

$$
\begin{aligned}
& -\left(\sum_{k=0}^{n-1} p^{\beta-k-1} f^{(k)}\right)(0) \ominus\left(-p^{\beta} \mathbf{L}[f(t)]\right) \\
& \quad=\left[\ell\left[\left({ }^{C} D_{a^{+}}^{\beta} \bar{f}\right)(x ; r), \ell\left({ }^{C} D_{a^{+}}^{\beta} f\right)(x ; r)\right]\right] .
\end{aligned}
$$

Thus, we obtain the following:

$$
\begin{gathered}
-\left(\sum_{k=0}^{n-1} p^{\beta-k-1} f^{(k)}\right)(0) \ominus\left(-p^{\beta} \mathbf{L}[f(t)]\right) \\
=\mathbf{L}\left[\left({ }^{C} D_{a^{+}}^{\beta} f\right)(x)\right],
\end{gathered}
$$

which completes the proof.

\section{Fractional Green's Functions}

In the following, we present the so-called fundamental solutions of fractional Green's functions for fuzzy fractional differential equations under Caputo's $\mathrm{H}$-differentiability. First we consider the nonhomogeneous fuzzy fractional differential equation of order $0<\beta<1$ together with nonhomogeneous initial condition

$$
\begin{gathered}
\left({ }^{C} D_{0^{+}}^{2 \beta} \Phi\right)(x) \oplus\left({ }^{C} D_{0^{+}}^{2 \beta}\right)(x) \\
=\varphi[x ; \Phi(x)], \quad 0<\beta<1, \\
\Phi(0)=f(x) \in \mathbb{E},
\end{gathered}
$$

where $t>0$. Fractional Green's functions for case so-called the classical Green's functions, that is, considering $\varphi(x)=$ $\delta(x)$ and $\Phi(0)=\widetilde{0}$, are discussed.

4.1. Determining Algebraic Solutions. The algebraic solutions of fuzzy fractional differential equations have been investigated under Caputo's H-differentiability. We provided the fuzzy Laplace transform to obtain the solutions of FFDE (42). By taking Laplace transform on the both sides of (42), we get

$$
\mathbf{L}\left[\left({ }^{C} D_{0^{+}}^{2 \beta} \Phi\right)(x) \oplus\left({ }^{C} D_{0^{+}}^{\beta} \Phi\right)(x)\right]=\mathbf{L}[\varphi(x, \Phi(x))] .
$$

After that, based on the types of Caputo's H-differentiability we have the following cases.

Case I. Let us consider that $D \Phi(x)$ is a ${ }^{C}[(\mathrm{i})-\beta]$-differentiable function; then (43) is extended based on the its lower and upper functions as follows:

$$
\begin{aligned}
\ell[\underline{\varphi}(x, \Phi(x) ; r)]= & p^{2 \beta} \ell[\underline{\Phi}(x, r)]-p^{2 \beta-1} \underline{\Phi}(0, r) \\
& +p^{\beta} \ell[\underline{\Phi}(x, r)]-p^{\beta-1} \Phi(0, r), \\
\ell[\bar{\varphi}(x, \Phi(x) ; r)]= & p^{2 \beta} \ell[\Phi(x, r)]-p^{2 \beta-1} \bar{\Phi}(0, r) \\
& +p^{\beta} \ell[\bar{\Phi}(x, r)]-p^{\beta-1} \bar{\Phi}(0, r),
\end{aligned}
$$

where

$$
\begin{array}{r}
\underline{\varphi}(x, \Phi(x) ; r)=\min \{\varphi(x, u) \mid \\
u \in[\underline{\Phi}(x ; r), \bar{\Phi}(x ; r)]\}, \\
0 \leq r \leq 1, \\
\bar{\varphi}(x, \Phi(x) ; r)=\max \{\varphi(x, u) \mid \\
u \in[\underline{\Phi}(x ; r), \bar{\Phi}(x ; r)]\}, \\
0 \leq r \leq 1 .
\end{array}
$$

In order to solve the linear system (44), for simplify we assume that

$$
\begin{array}{ll}
\ell[\Phi(x ; r)]=H_{1}(p ; r), \quad 0 \leq r \leq 1, \\
\ell[\bar{\Phi}(x ; r)]=K_{1}(p ; r), \quad 0 \leq r \leq 1,
\end{array}
$$


where $H_{1}(p ; r)$ and $K_{1}(p ; r)$ are solutions of system (44). Then, by using the inverse Laplace transform, $\Phi(x ; r)$ and $\bar{\Phi}(x ; r)$ are computed as follows:

$$
\begin{aligned}
\Phi(x, r) & =\ell^{-1}\left[H_{1}(p ; r)\right] \\
& =\underline{\Phi}(0 ; r) G_{1}(t)+\int_{0}^{t} G_{1}(t ; \tau) \underline{\varphi}(t-\tau ; r) d \tau, \\
& 0 \leq r \leq 1, \\
\Phi(x ; r) & =\ell^{-1}\left[K_{1}(p ; r)\right] \\
& =\bar{\Phi}(0 ; r) G_{1}(t)+\int_{0}^{t} G_{1}(t ; \tau) \bar{\varphi}(t-\tau ; r) d \tau, \\
& 0 \leq r \leq 1 .
\end{aligned}
$$

To explicit the corresponding Green's function, which is solution of the above relation satisfying the condition $\Phi(0)=$ $\widetilde{0}$ and taking $\varphi(x)=\delta(x)$, we use the linearity of the inverse Laplace transform to obtain

$$
G_{1}(t \mid 0)=\ell^{-1}[\Phi(x ; r)] .
$$

Case II. Let us consider that $D \Phi(x)$ is ${ }^{C}[(\mathrm{ii})-\beta]$ differentiable; then (43) can be written as follows:

$$
\begin{aligned}
\ell[\underline{\varphi}(x, \Phi(x) ; r)]= & p^{2 \beta} \ell[\underline{\Phi}(x, r)]-p^{2 \beta-1} \underline{\Phi}(0, r) \\
& +p^{\beta} \ell[\underline{\Phi}(x, r)]-p^{\beta-1} \Phi(0, r), \\
\ell[\bar{\varphi}(x, \Phi(x) ; r)]= & p^{2 \beta} \ell[\Phi(x, r)]-p^{2 \beta-1} \bar{\Phi}(0, r) \\
& +p^{\beta} \ell[\bar{\Phi}(x, r)]-p^{\beta-1} \bar{\Phi}(0, r),
\end{aligned}
$$

where

$$
\begin{array}{r}
\underline{\varphi}(x, \Phi(x) ; r)=\min \{\varphi(x, u) \mid u \in[\underline{\Phi}(x ; r), \bar{\Phi}(x ; r)]\}, \\
0 \leq r \leq 1, \\
\bar{\varphi}(x, \Phi(x) ; r)=\max \{\varphi(x, u) \mid u \in[\underline{\Phi}(x ; r), \bar{\Phi}(x ; r)]\}, \\
0 \leq r \leq 1 .
\end{array}
$$

Consequently, to solve the linear system (49), we set for simplify

$$
\begin{aligned}
& \ell[\Phi(x ; r)]=H_{2}(p ; r), \\
& \ell[\bar{\Phi}(x ; r)]=K_{2}(p ; r),
\end{aligned}
$$

where $H_{2}(p ; r)$ and $K_{2}(p ; r)$ are solutions of system (49). Then by making use of the inverse Laplace transform, $\Phi(x ; r)$ and $\bar{\Phi}(x ; r)$ are computed as follows:

$$
\begin{aligned}
\underline{\Phi}(x ; r)= & \ell^{-1}\left[H_{2}(p ; r)\right] \\
= & \underline{\Phi}(0 ; r) G_{2}(t) \\
& +\int_{0}^{t} G_{2}(t ; \tau) \underline{\varphi}(t-\tau ; r) d \tau, \\
\bar{\Phi}(x ; r)= & \ell^{-1}\left[K_{2}(p ; r)\right] \\
= & \bar{\Phi}(0 ; r) G_{2}(t) \\
& +\int_{0}^{t} G_{2}(t ; \tau) \bar{\varphi}(t-\tau ; r) d \tau, \\
& 0 \leq r \leq 1 .
\end{aligned}
$$

To show the corresponding Green's function, which is solution of (42) satisfying the condition $\Phi(0)=\widetilde{0}$ and taking $\varphi(x)=\delta(x)$, we use the linearity of the inverse Laplace transform to obtain

$$
G_{2}(t \mid 0)=\ell^{-1}[\Phi(x ; r)] .
$$

\section{Examples}

In this section, we consider several examples, and we find explicit expressions for fractional Green's functions, including some versions of the fuzzy harmonic oscillator equations and fuzzy relaxation equation under Caputo's Hdifferentiability.

Example 1 (Green's function for fuzzy damped harmonic oscillator). Let us examine the following FFDE:

$$
\begin{gathered}
a \odot\left({ }^{C} D_{0^{+}}^{2 \beta} \Phi\right)(x)+b \odot\left({ }^{C} D_{0^{+}}^{\beta} \Phi\right)(x) \\
=\lambda w^{2} \odot \Phi(x)+\varphi(x), \quad 0<\beta<1, \\
\Phi(0) \in \mathbb{E},
\end{gathered}
$$

where $a, b$ are real positive constants and $w$ is the frequency of the harmonic oscillator. We will solve this example according to the two following cases for $\lambda \in \mathbb{R}$.

Case I. Suppose that $\lambda \in \mathbb{R}^{+}=(0, \infty)$; then taking Laplace transform on the both sides of above equation, we have

$$
\begin{gathered}
\mathbf{L}\left[a \odot\left({ }^{C} D_{0^{+}}^{2 \beta} \Phi\right)(x)+b \odot\left({ }^{C} D_{0^{+}}^{\beta} \Phi\right)(x)\right] \\
=\mathbf{L}\left[\lambda w^{2} \odot \Phi(x)+\varphi(x)\right] .
\end{gathered}
$$


Using ${ }^{C}[(i)-\beta]$-differentiability, we get

$$
\begin{aligned}
\lambda w^{2} \ell & {[\underline{\Phi}(x ; r)]+\ell[\underline{\varphi}(x ; r)] } \\
= & a p^{2 \beta} \ell[\underline{\Phi}(x, r)]-a p^{2 \beta-1} \underline{\Phi}(0, r) \\
& +b p^{\beta} \ell[\underline{\Phi}(x, r)]-b p^{\beta-1} \underline{\Phi}(0, r), \\
\lambda w^{2} \ell & {[\bar{\Phi}(x ; r)]+\ell[\bar{\varphi}(x ; r)] } \\
= & a p^{2 \beta} \ell[\bar{\Phi}(x, r)]-a p^{2 \beta-1} \bar{\Phi}(0, r) \\
& +b p^{\beta} \ell[\bar{\Phi}(x, r)]-b p^{\beta-1} \bar{\Phi}(0, r) .
\end{aligned}
$$

Then, after some calculations, we obtain the following:

$$
\begin{aligned}
&\left(a p^{2 \beta}+b p^{\beta}-\lambda w^{2}\right) \ell[\underline{\Phi}(x ; r)] \\
&=\left(a p^{2 \beta-1}+b p^{\beta-1}\right) \underline{\Phi}(0 ; r)+\ell[\underline{\varphi}(x ; r)], \\
&\left(a p^{2 \beta}+b p^{\beta}-\lambda w^{2}\right) \ell[\bar{\Phi}(x ; r)] \\
&=\left(a p^{2 \beta-1}+b p^{\beta-1}\right) \bar{\Phi}(0 ; r)+\ell[\bar{\varphi}(x ; r)] .
\end{aligned}
$$

Consequently, taking the inverse of Laplace on both sides of (57) we have

$$
\begin{aligned}
\underline{\Phi}(x ; r)=\underline{\Phi}(0 ; r) \ell^{-1} & \\
& \times\left[\frac{\left(a p^{2 \beta-1}+b p^{\beta-1}\right) \underline{\Phi}(0 ; r)+\ell[\underline{\varphi}(x ; r)]}{a p^{2 \beta}+b p^{\beta}-\lambda w^{2}}\right], \\
& 0 \leq r \leq 1,
\end{aligned}
$$$$
\bar{\Phi}(x ; r)=\bar{\Phi}(0 ; r) \ell^{-1}
$$$$
\times\left[\frac{\left(a p^{2 \beta-1}+b p^{\beta-1}\right) \bar{\Phi}(0 ; r)+\ell[\bar{\varphi}(x ; r)]}{a p^{2 \beta}+b p^{\beta}-\lambda w^{2}}\right],
$$$$
0 \leq r \leq 1 \text {. }
$$

Finally, we determine the solution of FFDE as follows:

$$
\begin{aligned}
\underline{\Phi}(x ; r)= & \underline{\Phi}(0 ; r) \sum_{j=0}^{\infty}\left(\frac{-b}{a}\right)^{j} x^{\beta j} E_{2 \beta, \beta_{j}+1}^{(j+1)}\left(\frac{\lambda w^{2}}{a} x^{2 \beta}\right) \\
& +\frac{b}{a} x^{\beta} E_{2 \beta, \beta_{j}+\beta+1}^{(j+1)}\left(\frac{\lambda w^{2}}{a} x^{2 \beta}\right) \\
& +\frac{1}{a} \sum_{j=0}^{\infty}\left(\frac{-b}{a}\right)^{j} \int_{0}^{x} \underline{\varphi}(t-y ; r) y^{\beta j+2 \beta-1} E_{2 \beta, \beta_{j}+2 \beta}^{(j+1)} \\
& \times\left(\frac{\lambda w^{2}}{a} y^{2 \beta}\right) d y, \quad 0 \leq r \leq 1,
\end{aligned}
$$

$$
\begin{aligned}
\bar{\Phi}(x ; r)= & \bar{\Phi}(0 ; r) \sum_{j=0}^{\infty}\left(\frac{-b}{a}\right)^{j} x^{\beta j} E_{2 \beta, \beta_{j}+1}^{(j+1)}\left(\frac{\lambda w^{2}}{a} x^{2 \beta}\right) \\
& +\frac{b}{a} x^{\beta} E_{2 \beta, \beta_{j}+\beta+1}^{(j+1)}\left(\frac{\lambda w^{2}}{a} x^{2 \beta}\right) \\
& +\frac{1}{a} \sum_{j=0}^{\infty}\left(\frac{-b}{a}\right)^{j} \int_{0}^{x} \bar{\varphi}(t-y ; r) y^{\beta j+2 \beta-1} E_{2 \beta, \beta_{j}+2 \beta}^{(j+1)} \\
& \times\left(\frac{\lambda w^{2}}{a} y^{2 \beta}\right) d y, \quad 0 \leq r \leq 1 .
\end{aligned}
$$

To explicit the corresponding Green's function under ${ }^{C}[(\mathrm{i})-$ $\beta$ ]-differentiability, which is solution of (54) satisfying the condition $\Phi(0)=\widetilde{0}$ and taking $\varphi(x)=\delta(x)$, we use the linearity of the inverse Laplace transform to obtain

$$
\begin{aligned}
G_{1}(x \mid 0) & \equiv \ell^{-1}[\ell[\Phi(x ; r)]] \\
& =\frac{1}{a} \sum_{j=0}^{\infty}\left(\frac{-b}{a}\right)^{j} t^{\beta j+2 \beta-1} E_{2 \beta, \beta_{j}+2 \beta}^{(j+1)}\left(\frac{\lambda w^{2}}{a} t^{2 \beta}\right) .
\end{aligned}
$$

On the other hand, taking $\varphi(x)=\widetilde{0}$, we have a homogeneous fuzzy fractional differential equation whose solution under ${ }^{C}[(\mathrm{i})-\beta]$-H-differentiability is given by

$$
\begin{aligned}
\underline{\Phi}(x ; r)= & \Phi(0 ; r) \sum_{j=0}^{\infty}\left(\frac{-b}{a}\right)^{j} x^{\beta j} E_{2 \beta, \beta_{j}+1}^{(j+1)}\left(\frac{\lambda w^{2}}{a} x^{2 \beta}\right) \\
& +\frac{b}{a} x^{\beta} E_{2 \beta, \beta_{j}+\beta+1}^{(j+1)}\left(\frac{\lambda w^{2}}{a} x^{2 \beta}\right), \quad 0 \leq r \leq 1, \\
\bar{\Phi}(x ; r)= & \bar{\Phi}(0 ; r) \sum_{j=0}^{\infty}\left(\frac{-b}{a}\right)^{j} x^{\beta j} E_{2 \beta, \beta_{j}+1}^{(j+1)}\left(\frac{\lambda w^{2}}{a} x^{2 \beta}\right) \\
& +\frac{b}{a} x^{\beta} E_{2 \beta, \beta_{j}+\beta+1}^{(j+1)}\left(\frac{\lambda w^{2}}{a} x^{2 \beta}\right), \quad 0 \leq r \leq 1 .
\end{aligned}
$$

Case II. If $\lambda \in \mathbb{R}^{-}=(-\infty, 0)$, then by using ${ }^{C}[(\mathrm{ii})-$ $\beta$ ]-differentiability and Theorem 16 , and their inverses, fractional Green's function will be obtained similar to (60).

Example 2 (Green's function for fuzzy the driven harmonic oscillator). Let us investigate the following FFDEs; namely,

$$
\begin{gathered}
\left({ }^{C} D_{0^{+}}^{2 \beta} \Phi\right)(x)=\lambda w^{2 \beta} \odot \Phi(x)+\varphi(x), \quad 0<\beta<1 \\
\Phi(0) \in \mathbb{E},
\end{gathered}
$$

with $b=0$ and $1 / 2<\beta<1$; the fuzzy fractional differential equation associated with the driven harmonic oscillator is obtained. We obtain the same result taking $b=0, a=1$ and solve this example according to the two following cases for $\lambda \in \mathbb{R}$. 
Case I. Suppose that $\lambda \in \mathbb{R}^{+}=(0,+\infty)$; then taking Laplace transform on the both sides of above equation, we have the following:

$$
\mathbf{L}\left[\left({ }^{C} D_{a^{+}}^{2 \beta} \Phi\right)(x)\right]=\mathbf{L}\left(\lambda w^{2 \beta} \odot \Phi(x)+\varphi(x)\right) .
$$

Using ${ }^{C}[(i)-\beta]$-differentiability, we obtain

$$
\begin{aligned}
\lambda w^{2 \beta} \ell & {[\underline{\Phi}(x ; r)]+\ell[\underline{\varphi}(x ; r)] } \\
& =p^{2 \beta} \ell[\underline{\Phi}(x, r)]-p^{2 \beta-1} \underline{\Phi}(0, r), \\
\lambda w^{2 \beta} \ell & {[\bar{\Phi}(x ; r)]+\ell[\bar{\varphi}(x ; r)] } \\
& =p^{2 \beta} \ell[\bar{\Phi}(x, r)]-p^{2 \beta-1} \underline{\Phi}(0, r) .
\end{aligned}
$$

Then, after some manipulations the following result is reported:

$$
\begin{aligned}
& \left(p^{2 \beta}-\lambda w^{2 \beta}\right) \ell[\underline{\Phi}(x ; r)]=p^{2 \beta-1} \underline{\Phi}(0, r)+\ell[\underline{\varphi}(x ; r)], \\
& \left(p^{2 \beta}-\lambda w^{2 \beta}\right) \ell[\bar{\Phi}(x ; r)]=p^{2 \beta-1} \bar{\Phi}(0, r)+\ell[\bar{\varphi}(x ; r)] .
\end{aligned}
$$

By taking inverse of Laplace on the both sides of (75) we have

$$
\begin{array}{r}
\underline{\Phi}(x ; r)=\underline{\Phi}(0 ; r) \ell^{-1}\left[\frac{p^{2 \beta-1} \underline{\Phi}(0, r)+\ell[\underline{\varphi}(x ; r)]}{p^{2 \beta}-\lambda w^{2 \beta}}\right], \\
0 \leq r \leq 1, \\
\Phi(x ; r)=\bar{\Phi}(0 ; r) \ell^{-1}\left[\frac{p^{2 \beta-1} \underline{\Phi}(0, r)+\ell[\bar{\varphi}(x ; r)]}{p^{2 \beta}-\lambda w^{2 \beta}}\right], \\
0 \leq r \leq 1 .
\end{array}
$$

Finally, we determine the solution of FFDE as follows:

$$
\begin{aligned}
\underline{\Phi}(x ; r)= & \underline{\Phi}(0 ; r) E_{2 \beta}\left(\lambda w^{2 \beta} x^{2 \beta}\right) \\
& +\int_{0}^{x} \underline{\varphi}(t-y ; r) y^{2 \beta-1} E_{2 \beta, 2 \beta}\left(\lambda w^{2 \beta} y^{2 \beta}\right) d y, \\
\bar{\Phi}(x ; r)= & 0 \leq r \leq 1, \\
& +\int_{0}^{x} \bar{\varphi}(0 ; r) E_{2 \beta}\left(\lambda w^{2 \beta} x^{2 \beta}\right) \\
& \\
& 0 \leq r \leq 1) y^{2 \beta-1} E_{2 \beta, 2 \beta}\left(\lambda w^{2 \beta} y^{2 \beta}\right) d y,
\end{aligned}
$$

To show the corresponding Green's function under ${ }^{C}[(\mathrm{i})-$ $\beta]$-differentiability, which is solution of (62) satisfying the condition $\Phi(0)=\widetilde{0}$ and taking $\varphi(x)=\delta(x)$, we use the linearity of the inverse Laplace transform to obtain

$$
G_{1}(x \mid 0) \equiv x^{2 \beta-1} E_{2 \beta, 2 \beta}\left(\lambda w^{2 \beta} x^{2 \beta}\right) .
$$

In the case $\beta=1$, we report

$$
G_{1}(x \mid 0)=x E_{2,2}\left(\lambda w^{2} x^{2}\right)
$$

which is the classic Green's function associated with fuzzy the driven harmonic oscillator.

Taking $\varphi(x)=\widetilde{0}$, we have a homogeneous fuzzy fractional differential equation whose solution under ${ }^{C}[(i)-\beta]-\mathrm{H}$ differentiability is given by

$$
\begin{array}{ll}
\underline{\Phi}(x ; r)=\underline{\Phi}(0 ; r) E_{2 \beta}\left(\lambda w^{2 \beta} x^{2 \beta}\right), & 0 \leq r \leq 1, \\
\Phi(x ; r)=\bar{\Phi}(0 ; r) E_{2 \beta}\left(\lambda w^{2 \beta} x^{2 \beta}\right), & 0 \leq r \leq 1 .
\end{array}
$$

For $\beta=1$, we get

$$
\begin{array}{ll}
\underline{\Phi}(x ; r)=\underline{\Phi}(0 ; r) \cos w t, & 0 \leq r \leq 1, \\
\bar{\Phi}(x ; r)=\bar{\Phi}(0 ; r) \cos w t, & 0 \leq r \leq 1 .
\end{array}
$$

Case II. Let us suppose that $\lambda \in \mathbb{R}^{-}=(-\infty, 0)$; then using ${ }^{C}[(\mathrm{ii})-\beta]$-differentiability and Theorem 16 and their inverses, fractional Green's function will be obtained similar to (68).

Example 3 (Green's functions for fuzzy fractional relaxation equation). The next step is to consider the following FFDE:

$$
\begin{gathered}
b \odot\left({ }^{C} D_{0^{+}}^{\beta} \Phi\right)(x)=\lambda w^{2} \odot \Phi(x)+\varphi(x), \\
0<\beta<1, \\
\Phi(0) \in \mathbb{E},
\end{gathered}
$$

where $a=0$ in (54) and $0<\beta<1$. We solve this example according to the two following cases corresponding to $\lambda \in \mathbb{R}$.

Case I. Suppose that $\lambda \in \mathbb{R}^{+}=(0,+\infty)$; then taking Laplace transform on the both sides of above equation, we have

$$
\mathbf{L}\left[b \odot\left({ }^{C} D_{a^{+}}^{\beta} \Phi\right)(x)\right]=\mathbf{L}\left[\lambda w^{2} \odot \Phi(x)+\varphi(x)\right] .
$$

Using ${ }^{C}[(i)-\beta]$-differentiability, we get

$$
\begin{aligned}
\lambda w^{\beta} \ell & {[\underline{\Phi}(x ; r)]+\ell[\underline{\varphi}(x ; r)] } \\
& =b p^{\beta} \ell[\underline{\Phi}(x, r)]-b p^{\beta-1} \underline{\Phi}(0, r), \\
\lambda w^{\beta} \ell & {[\bar{\Phi}(x ; r)]+\ell[\bar{\varphi}(x ; r)] } \\
& =b p^{\beta} \ell[\bar{\Phi}(x, r)]-b p^{\beta-1} \underline{\Phi}(0, r) .
\end{aligned}
$$

Then, after some manipulations we get the following:

$$
\begin{aligned}
& \left(b p^{\beta}-\lambda w^{2}\right) \ell[\underline{\Phi}(x ; r)]=b p^{\beta-1} \underline{\Phi}(0, r)+\ell[\underline{\varphi}(x ; r)], \\
& \left(b p^{\beta}-\lambda w^{2}\right) \ell[\bar{\Phi}(x ; r)]=b p^{\beta-1} \bar{\Phi}(0, r)+\ell[\bar{\varphi}(x ; r)] .
\end{aligned}
$$


By taking inverse of Laplace on the both sides of (75) we have

$$
\begin{array}{r}
\underline{\Phi}(x ; r)=\underline{\Phi}(0 ; r) \ell^{-1}\left[\frac{b p^{\beta-1} \underline{\Phi}(0, r)+\ell[\underline{\varphi}(x ; r)]}{b p^{\beta}-\lambda w^{2}}\right], \\
0 \leq r \leq 1, \\
\Phi(x ; r)=\bar{\Phi}(0 ; r) \ell^{-1}\left[\frac{b p^{\beta-1} \Phi(0, r)+\ell[\bar{\varphi}(x ; r)]}{b p^{\beta}-\lambda w^{2}}\right], \\
0 \leq r \leq 1 .
\end{array}
$$

Thus, we determine the solution of FFDE as follows:

$$
\begin{aligned}
& \underline{\Phi}(x ; r)= \underline{\Phi}(0 ; r) E_{\beta}\left(\frac{\lambda w^{2}}{b} x^{\beta}\right) \\
&+\frac{1}{b} \int_{0}^{x} \underline{\varphi}(t-y ; r) y^{\beta-1} E_{\beta, \beta}\left(\frac{\lambda w^{2}}{b} y^{\beta}\right) d y, \\
& 0 \leq r \leq 1, \\
& \Phi(x ; r)=\bar{\Phi}(0 ; r) E_{\beta}\left(\frac{\lambda w^{2}}{b} x^{\beta}\right) \\
&+ \frac{1}{b} \int_{0}^{x} \bar{\varphi}(t-y ; r) y^{\beta-1} E_{\beta, \beta}\left(\frac{\lambda w^{2}}{b} y^{\beta}\right) d y, \\
& 0 \leq r \leq 1 .
\end{aligned}
$$

Here, we obtain explicitly Green's functions under ${ }^{C}[(i)-\beta]$ differentiability, introducing $\varphi(x)=\delta(x)$ and $\Phi(0)=\widetilde{0}$; that is,

$$
G_{1}(x \mid 0) \equiv \frac{1}{b} x^{\beta-1} E_{\beta, \beta}\left(\frac{\lambda w^{\beta}}{b} x^{\beta}\right) .
$$

When $\beta=1$, we get the classic Green's function under strongly generalized differentiability

$$
G_{1}(x \mid 0)=\frac{1}{b} \exp \left(\frac{\lambda w^{2}}{b} x\right) .
$$

On the other hand, taking $\varphi(x)=\widetilde{0}$, we have a homogeneous fuzzy fractional differential equation whose solution under ${ }^{C}[(i)-\beta]$-H-differentiability is given by

$$
\begin{array}{ll}
\underline{\Phi}(x ; r)=\underline{\Phi}(0 ; r) E_{\beta}\left(\frac{\lambda w^{2}}{b} x^{\beta}\right), & 0 \leq r \leq 1, \\
\Phi(x ; r)=\bar{\Phi}(0 ; r) E_{\beta}\left(\frac{\lambda w^{2}}{b} x^{\beta}\right), & 0 \leq r \leq 1 .
\end{array}
$$

Finally, in the case $\beta=1$ we get

$$
\begin{array}{ll}
\Phi(x ; r)=\underline{\Phi}(0 ; r) \exp \left(\frac{\lambda w^{2}}{b} x\right), & 0 \leq r \leq 1, \\
\Phi(x ; r)=\Phi(0 ; r) \exp \left(\frac{\lambda w^{2}}{b} x\right), & 0 \leq r \leq 1 .
\end{array}
$$

Case II. Suppose that $\lambda \in \mathbb{R}^{-}=(-\infty, 0)$; then using ${ }^{C}[(i i)-\beta]$-differentiability and Theorem 16 , and their inverses, fractional Green's function will be obtained similar to (78).

Now, we provide some examples such that the obtained solutions coincide with the previously reported solution in integer case [37].

Example 4. For a special case, let us consider the following FFDE:

$$
\begin{gathered}
\left({ }^{C} D_{0^{+}}^{\beta} \Phi\right)(x)=\lambda \odot \Phi(x), \quad 0<\beta, x<1, \\
\Phi(0) \in \mathbb{E},
\end{gathered}
$$

where $y$ represents the number of radionuclides present in a given radioactive and $\lambda$ is a decay constant.

Case I. Suppose that $\lambda \in \mathbb{R}^{+}=(0,+\infty)$; then using ${ }^{C}[(\mathrm{i})-$ $\beta$ ]-differentiability and Theorem 16 , the solution is derived as follows:

$$
\begin{array}{ll}
\Phi(x ; r)=\Phi(0, r) E_{\beta, \beta}\left[\lambda x^{\beta}\right], & 0 \leq r \leq 1, \\
\Phi(x ; r)=\bar{\Phi}(0, r) E_{\beta, \beta}\left[\lambda x^{\beta}\right], & 0 \leq r \leq 1 .
\end{array}
$$

Case II. Suppose that $\lambda \in \mathbb{R}^{-}=(-\infty, 0)$; then using ${ }^{C}[($ ii $)-\beta]$-differentiability and Theorem 16 , the solution will be obtained similar to (83). For more details see Figures 1, 2, 3 , and 4 .

Example 5. Let us discuss the following FFDE:

$$
\begin{gathered}
\left({ }^{C} D_{0^{+}}^{\beta} \Phi\right)(x)=(-1) \odot \Phi(x)+x+1, \quad 0<\beta, x<1, \\
\Phi(0) \in \mathbb{E} .
\end{gathered}
$$

Then, using ${ }^{C}[($ ii) $-\beta]$-differentiability and Theorem 16 , the solution becomes as follows:

$$
\begin{aligned}
\underline{y}(x ; r)= & \underline{\Phi}(0, r) E_{\beta, \beta}\left[\lambda x^{\beta}\right] \\
& +\int_{0}^{x}(x-t)^{\beta-1} E_{\beta, \beta}\left[\lambda(x-t)^{\beta}\right](t+1) d t, \\
\bar{y}(x ; r)= & 0 \leq r \leq 1, \\
& +\int_{0}^{x}(0, r) E_{\beta, \beta}\left[\lambda x^{\beta}\right] \\
& \\
& 0 \leq r \leq 1 .
\end{aligned}
$$

For more details see Figures 5 and 6.

\section{Conclusion}

In this paper, the fractional Green's functions for the solutions of FFDEs by using the fuzzy Laplace transforms under 


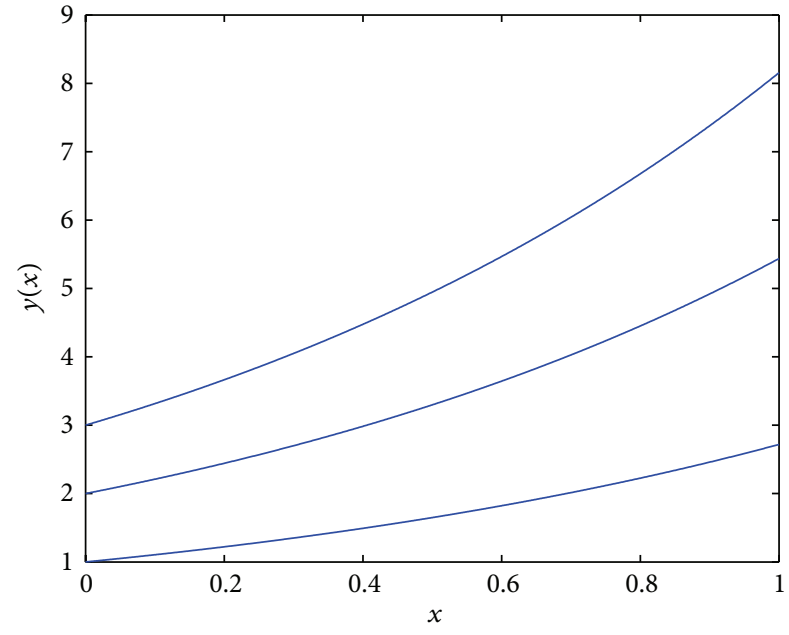

Figure 1: Solution of Example 4, Case I, $\beta=1$.

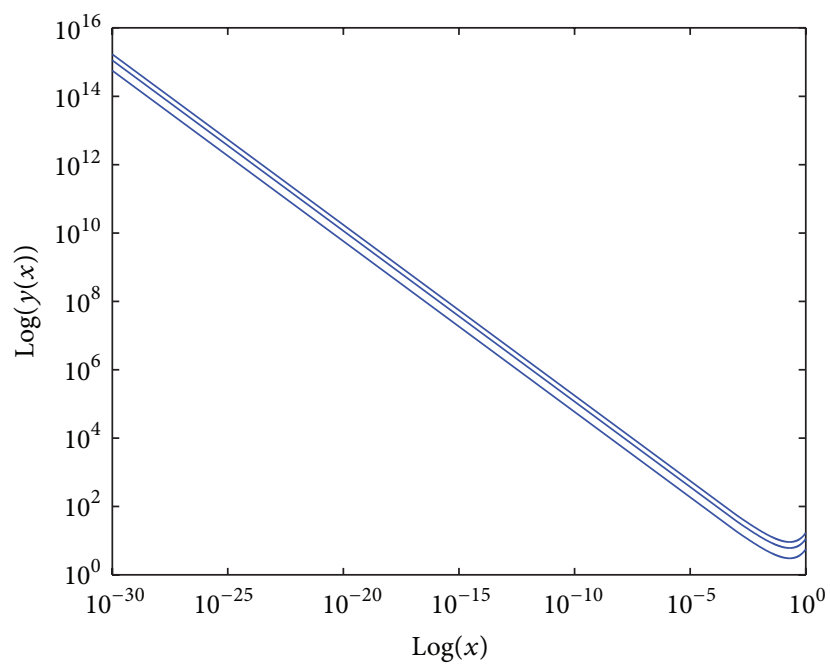

Figure 2: Solution of Example 4, Case I, $\beta=0.5$.

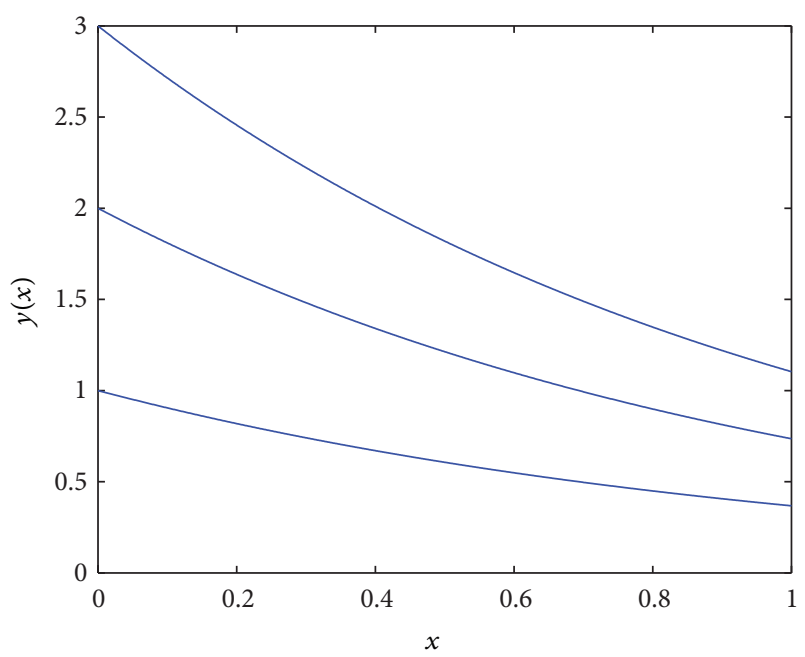

FIgURE 3: Solution of Example 4, Case II, $\beta=1$.

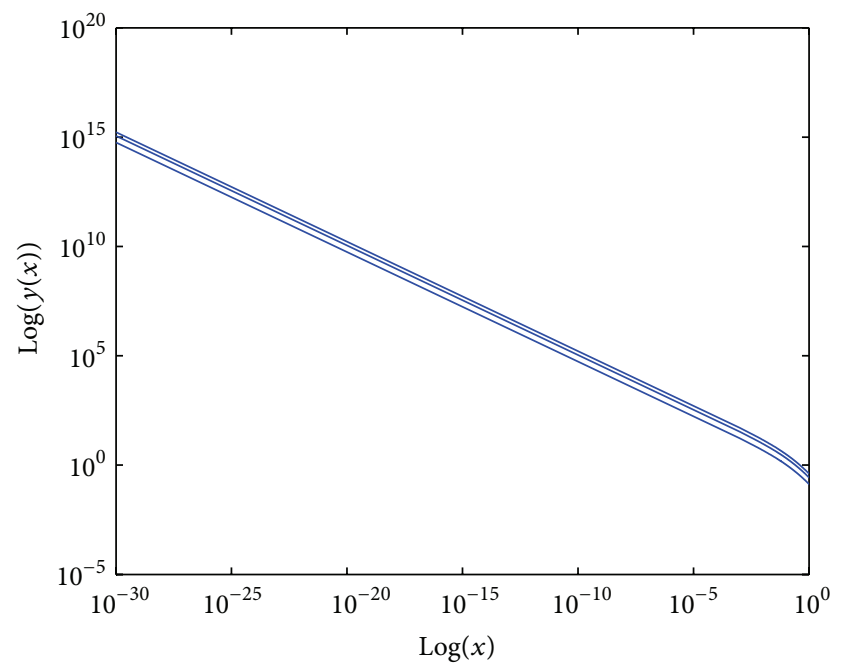

Figure 4: Solution of Example 4, Case II, $\beta=0.5$.

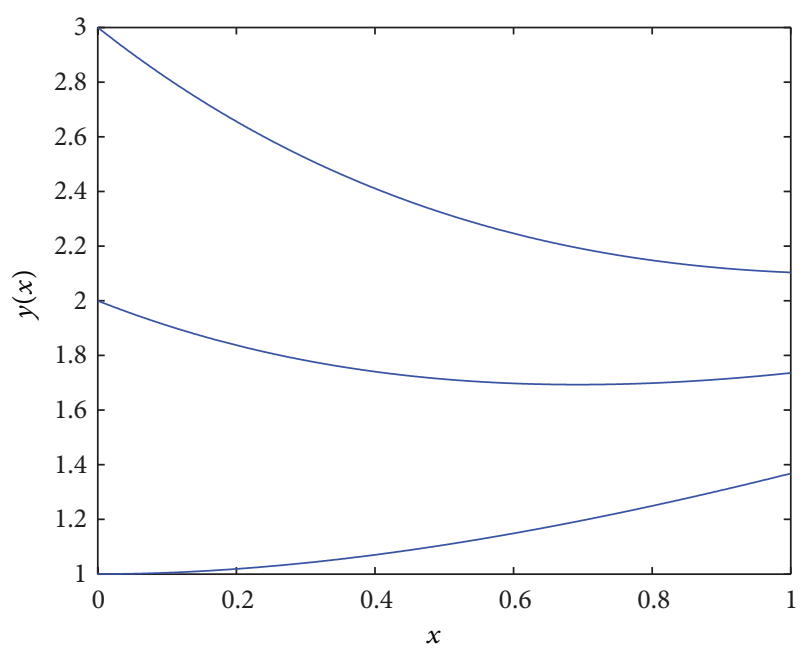

Figure 5: Solution of Example 5, $\beta=1$.

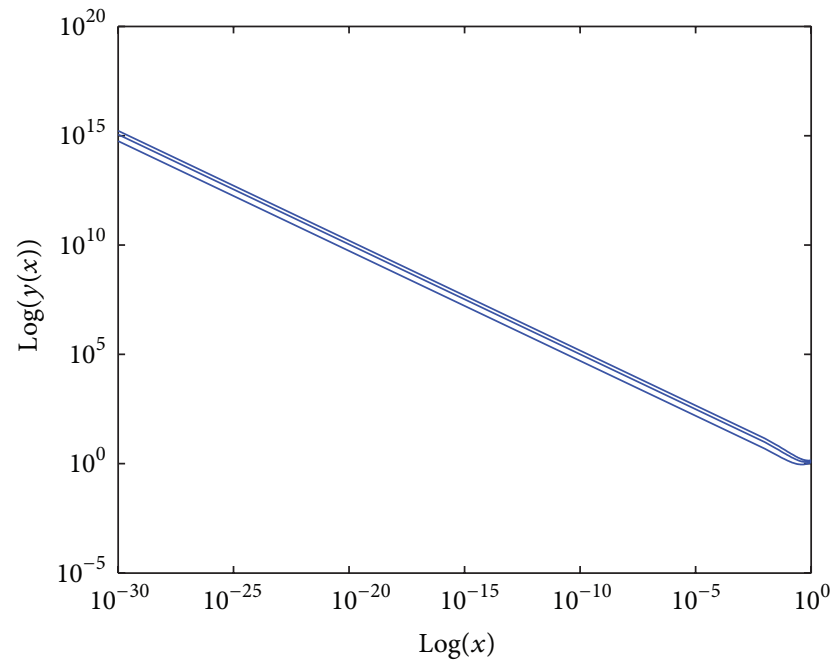

FIgURE 6: Solution of Example 5, $\beta=0.5$. 
Caputo's differentiability were applied. Some particular cases were also presented, involving some fuzzy versions of the harmonic oscillator. To this end, some needed concepts are given like the Caputo derivatives, fuzzy Laplace transforms, and their inverses, and so forth. For future research, we extend the boundary value problem for FDE [38] to the fractional case.

\section{Acknowledgments}

The authors thank the referees for careful reading and helpful suggestions on the improvement of the paper.

\section{References}

[1] A. Ahmadian, M. Suleiman, S. Salahshour, and D. Baleanu, "A Jacobi operational matrix for solving a fuzzy linear fractional differential equation," Advances in Difference Equations, vol. 2013, article 104, pp. 1-29, 2013.

[2] T. Allahviranloo and M. B. Ahmadi, "Fuzzy Laplace transforms," Soft Computing, vol. 14, no. 3, pp. 235-243, 2010.

[3] T. Allahviranloo, S. Salahshour, and S. Abbasbandy, "Explicit solutions of fractional differential equations with uncertainty," Soft Computing, vol. 16, no. 2, pp. 297-302, 2012.

[4] T. Allahviranloo, S. Abbasbandy, S. Salahshour, and A. Hakimzadeh, "A new method for solving fuzzy linear differential equations," Computing, vol. 92, no. 2, pp. 181-197, 2011.

[5] T. Allahviranloo and S. Salahshour, "A new approach for solving first order fuzzy differential equations," Communications in Computer and Information Science, vol. 81, part 5, part 6, pp. 522-531, 2010.

[6] J. Li, A. Zhao, and J. Yan, "The Cauchy problem of fuzzy differential equations under generalized differentiability," Fuzzy Sets and Systems, vol. 200, pp. 1-24, 2012.

[7] S. Salahshour and T. Allahviranloo, "Application of fuzzy differential transform method for solving fuzzy Volterra integral equations," Applied Mathematical Modelling, vol. 37, no. 3, pp. 1016-1027, 2013.

[8] S. Salahshour and A. Allahviranloo, "Applications of fuzzy Laplace transforms," Soft Computing, vol. 17, no. 1, pp. 145-178, 2013.

[9] A. Arara, M. Benchohra, N. Hamidi, and J. J. Nieto, "Fractional order differential equations on an unbounded domain," Nonlinear Analysis: Theory, Methods \& Applications, vol. 72, no. 2, pp. 580-586, 2010.

[10] Y. I. Babenko, Heat and Mass Transfer, Chemia, Leningrad, Russia, 1986.

[11] R. L. Bagley, "On the fractional order initial value problem and its engineering applications," in Fractional Calculus and Its Applications, K. Nishimoto, Ed., pp. 12-20, College of Engineering, Nihon University, Tokyo, Japan, 1990.

[12] H. Beyer and S. Kempfle, "Definition of physically consistent damping laws with fractional derivatives," Zeitschrift für Angewandte Mathematik und Mechanik, vol. 75, no. 8, pp. 623-635, 1995.

[13] K. Diethelm and N. J. Ford, "Analysis of fractional differential equations," Journal of Mathematical Analysis and Applications, vol. 265, no. 2, pp. 229-248, 2002.

[14] I. Podlubny, Fractional Differential Equations, vol. 198 of Mathematics in Science and Engineering, Academic Press, San Diego, Calif, USA, 1999.
[15] A. A. Kilbas, H. M. Srivastava, and J. J. Trujillo, Theory and Applications of Fractional Differential Equations, vol. 204 of North-Holland Mathematics Studies, Elsevier Science B.V., Amsterdam, The Netherland, 2006.

[16] V. Lakshmikantham, S. Leela, and J. Vasundhara Devi, Theory of Fractional Dynamic Systems, Cambridge Scientific, Cambridge, UK, 2009.

[17] V. Lakshmikantham and R. N. Mohapatra, Theory of Fuzzy Differential Equations and Inclusions, vol. 6 of Series in Mathematical Analysis and Applications, Taylor \& Francis, London, UK, 2003.

[18] V. Lakshmikantham and A. S. Vatsala, "Basic theory of fractional differential equations," Nonlinear Analysis: Theory, Methods \& Applications, vol. 69, no. 8, pp. 2677-2682, 2008.

[19] R. P. Agarwal, V. Lakshmikantham, and J. J. Nieto, "On the concept of solution for fractional differential equations with uncertainty," Nonlinear Analysis: Theory, Methods \& Applications, vol. 72, no. 6, pp. 2859-2862, 2010.

[20] B. Bede and S. G. Gal, "Generalizations of the differentiability of fuzzy-number-valued functions with applications to fuzzy differential equations," Fuzzy Sets and Systems, vol. 151, no. 3, pp. 581-599, 2005.

[21] B. Bede, I. J. Rudas, and A. L. Bencsik, "First order linear fuzzy differential equations under generalized differentiability," Information Sciences, vol. 177, no. 7, pp. 1648-1662, 2007.

[22] I. Perfilieva, "Fuzzy transforms: theory and applications," Fuzzy Sets and Systems, vol. 157, no. 8, pp. 993-1023, 2006.

[23] I. Perfilieva, H. De Meyer, B. De Baets, and D. Plšková, “Cauchy problem with fuzzy initial condition and its approximate solution with the help of fuzzy transform," in Proceedings of IEEE International Conference on Fuzzy Systems (FUZZ '08), pp. 2285-2290, June 2008.

[24] S. Salahshour, T. Allahviranloo, S. Abbasbandy, and D. Baleanu, "Existence and uniqueness results for fractional differential equations with uncertainty," Advances in Difference Equations, vol. 2012, article 112, pp. 1-12, 2012.

[25] M. Mazandarani and A. V. Kamyad, "Modified fractional Euler method for solving fuzzy fractional initial value problem," Communications in Nonlinear Science and Numerical Simulation, vol. 18, no. 1, pp. 12-21, 2013.

[26] R. P. Agarwal, S. Arshad, D. O’Regan, and V. Lupulescu, "Fuzzy fractional integral equations under compactness type condition," Fractional Calculus and Applied Analysis, vol. 15, no. 4, pp. 572-590, 2012.

[27] J. Xu, Z. Liao, and Z. Hu, "A class of linear differential dynamical systems with fuzzy initial condition," Fuzzy Sets and Systems, vol. 158, no. 21, pp. 2339-2358, 2007.

[28] M. Friedman, M. Ma, and A. Kandel, "Numerical solutions of fuzzy differential and integral equations," Fuzzy Sets and Systems, vol. 106, no. 1, pp. 35-48, 1999.

[29] M. Ma, M. Friedman, and A. Kandel, "Numerical solutions of fuzzy differential equations," Fuzzy Sets and Systems, vol. 105, no. 1, pp. 133-138, 1999.

[30] H.-J. Zimmermann, Fuzzy Set Theory and Its Applications, Kluwer Academic Publishers, Boston, Mass, USA, 2nd edition, 1992.

[31] M. L. Puri and D. A. Ralescu, "Fuzzy random variables," Journal of Mathematical Analysis and Applications, vol. 114, no. 2, pp. 409-422, 1986.

[32] H.-C. Wu, "The improper fuzzy Riemann integral and its numerical integration," Information Sciences, vol. 111, no. 1-4, pp. 109-137, 1998. 
[33] G. A. Anastassiou, Fuzzy Mathematics: Approximation Theory, vol. 251 of Studies in Fuzziness and Soft Computing, Springer, Berlin, Germany, 2010.

[34] G. A. Anastassiou, Intelligent Mathematics: Computational Analysis, vol. 5 of Intelligent Systems Reference Library, Springer, Berlin, Germany, 2011.

[35] S. Salahshour, T. Allahviranloo, and S. Abbasbandy, "Solving fuzzy fractional differential equations by fuzzy Laplace transforms," Communications in Nonlinear Science and Numerical Simulation, vol. 17, no. 3, pp. 1372-1381, 2012.

[36] Y. Chalco-Cano and H. Román-Flores, "On new solutions of fuzzy differential equations," Chaos, Solitons and Fractals, vol. 38, no. 1, pp. 112-119, 2008.

[37] A. Khastan, J. J. Nieto, and R. Rodríguez-López, "Variation of constant formula for first order fuzzy differential equations," Fuzzy Sets and Systems, vol. 177, pp. 20-33, 2011.

[38] A. Khastan, J. J. Nieto, and R. Rodríguez-López, "Periodic boundary value problems for first-order linear differential equations with uncertainty under generalized differentiability," Information Sciences, vol. 222, pp. 544-558, 2013. 


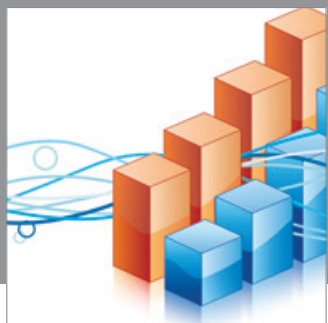

Advances in

Operations Research

mansans

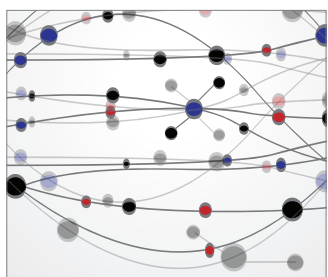

The Scientific World Journal
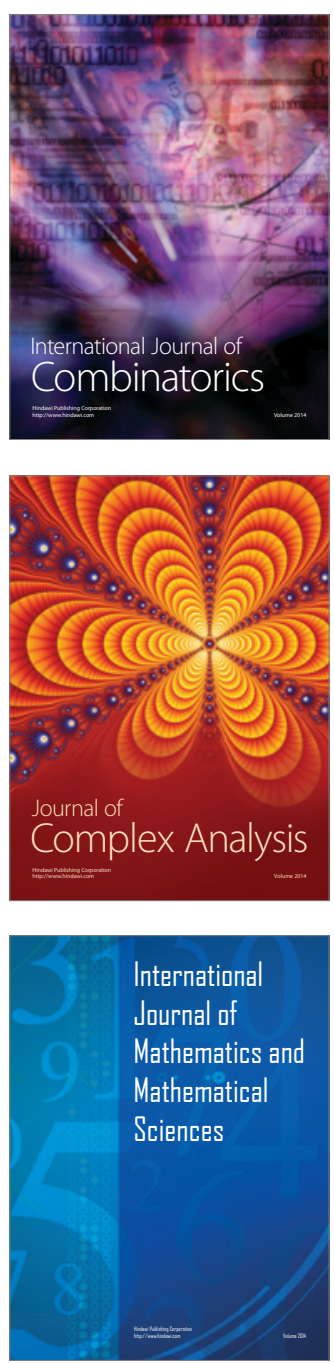
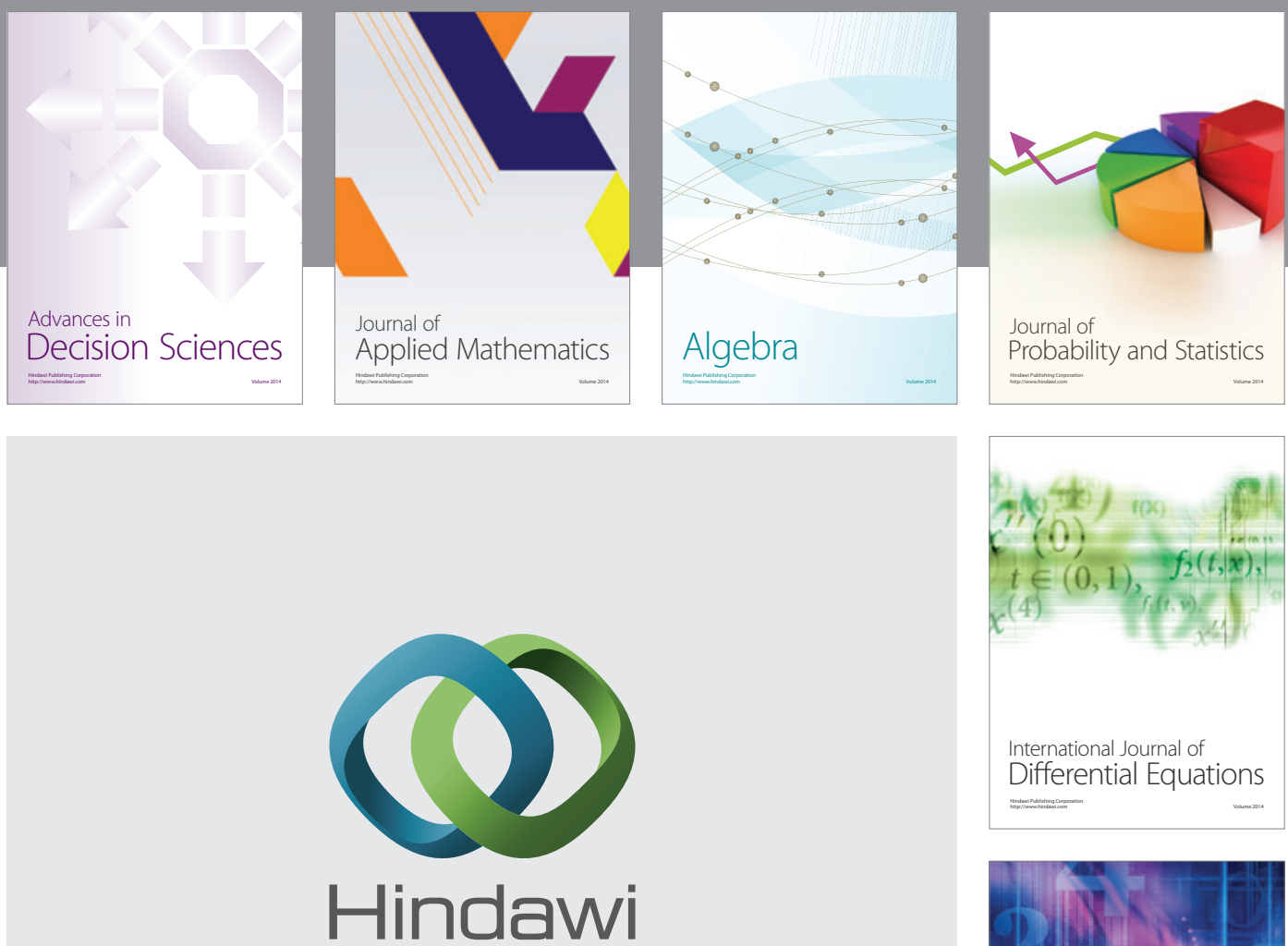

Submit your manuscripts at http://www.hindawi.com
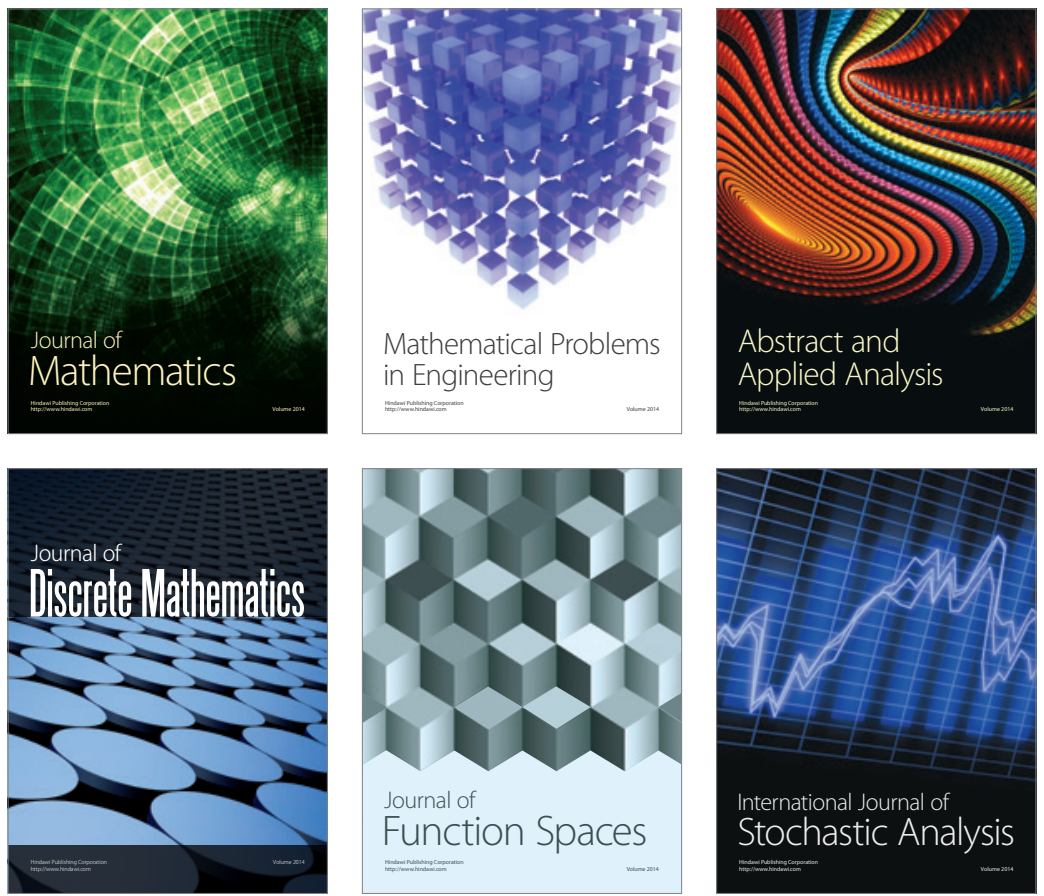

Journal of

Function Spaces

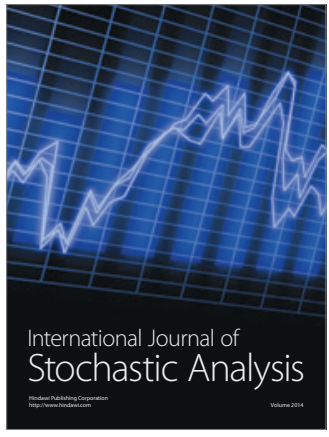

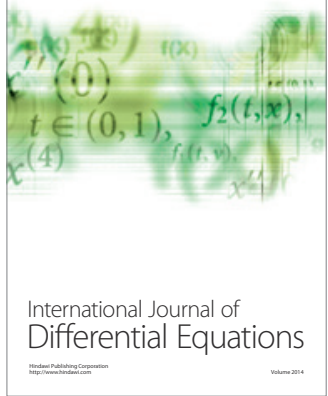
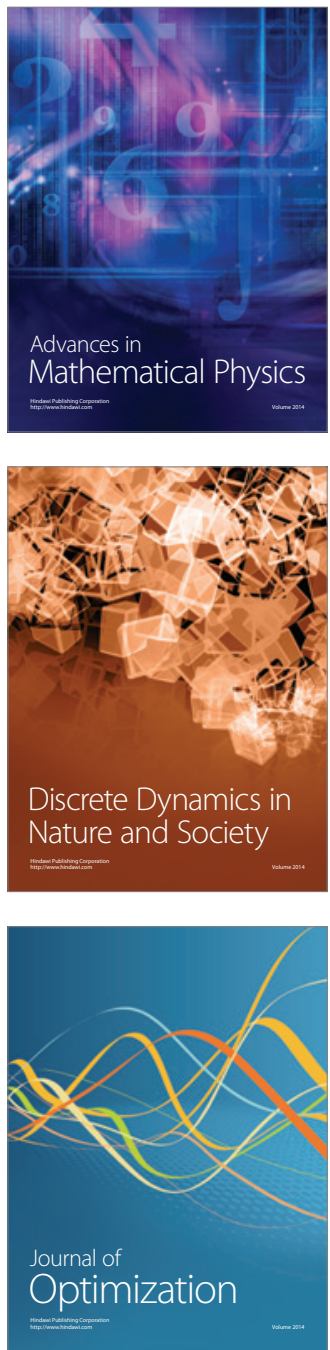